\title{
Contemporary Media Forum: Books, Films, and Audiocassettes
}

\section{EVERYTHING YOU'VE ALWAYS WANTED TO KNOW ABOUT PTSD, TRAUMATHERAPY AND TRAUMASCIENCE*}

\author{
A Clinical Handbook/Practical Therapist Manual: For Assessing and \\ Treating Adults with Post-Traumatic Stress Disorder (PTSD). Don- \\ ald Meichenbaum. Ontario, Canada: Institute Press, 1995, 600 pages, \\ $\$ 45.00$ US.
}

Not only does this highly clinical volume carry the credibility to serve as gateway to traumascience for the novice, but its power to spark new creativity and insights will inspire and guide even the seasoned clinician. This volume, $A$ Clinical Handbook/Practical Therapist Manual, is written by a giant in the field of clinical practice and clinical research. Internationally known for his clinical contributions in cognitive-behavior modification, stress inoculation training, and treatment adherence, Dr. Donald Meichenbaum, during the past seven years, has focused like a laser on the assessment and treatment of children, adolescents, and adults with post-traumatic stress disorder (PTSD) and allied conditions. This book in an outgrowth of his many professional experiences in traumatology, to include consultations on PTSD in adult psychiatric inpatients, patients on dual diagnosis units (PTSD and alcohol), traumatized children and youths in residential settings, and on children in shelters for children taken away from their parents because of sexual abuse.

Additionally, he has consulted in the areas of hostage-taking, school shooting, police shooting, in debriefing of victims of natural and technological disasters, and in the application of his stress inoculation training methods to military personnel (e.g., United Nations peace-keepers). The author's experience in training, his major editorial activities (as Editor of

*Well, almost everything! 
the Plenum Stress and Coping Book Series), private practice with traumatized individuals, and 30 years of doing. clinical work rounds out his experience to achieve the credibility essential to write and publish this Handbook/Manual.

This volume integrates the most exciting and clinically innovative, science-grounded techniques and procedures in the field of traumatology today. It even illuminates old concepts in new ways, as it takes the reader down two fundamental paths-one to familiarity and tradition, the other to innovation and creativity. After horrific events so many victims find themselves with broken affective, volitional, and behavioral parts, and because of pervasive biopsychobehavioral changes, "choose" to wear ill-fitted casts, bandages, and crutches. Clinicians who put into practice the integrated principles and guidelines found in the Handbook/Manual may find new ways to help clients make better choices over the "ill-fitted casts, bandages, and crutches" they felt "compelled" to wear in the past. The author skillfully brings together the works of many pioneering clinicians and clinical scientists, to include the works of A. Burgess, J. O. Brende, A. Egendorf, M. Friedman, C. R. Figley, J. Fairbanks, B. Green, M. Horowitz, T. Keene, L. Kolb, R. J. Lifton, J. Lindy, E. R. Parson, B. Raphael, C. Shatan, L. Terr, J. Wilson, and the many recent contributors whose writings have propelled traumatherapy and traumascience forward.

The major focus of the volume is the assessment and treatment of adults who have been psychologically assailed by a variety of traumatizing stimuli originating in such untoward events as rape, domestic violence and spousal abuse, child sexual abuse, incest, war, the Holocaust, torture, natural and human-engineered disasters, automobile accidents, and crime. The author, Dr. Donald Meichenbaum of the University of Waterloo, Canada, compiles a rich and significant body of relevant literature in psychotraumatology, and provide a practical "user-friendly" clinical guidebook on PTSD. His objective is to assist clinicians in the quest to become "more expert" in the field. He is aware that the volume cannot fully absorb the entire explosion of knowledge within psychotraumatology, but the Handbook/Manual is a gigantic effort in the right direction. The author plans to publish annual updates on PTSD, which he will make available to those who own the volume.

As the most important teaching manual of its kind to ever appear on the scene, this volume is encyclopedic in contents and scope, and is basically a useful reference guide for both the clinical and scientific traumatologist. It covers the origins of PTSD ("traumagenesis"), the various trauma subpopulations ("traumadiversity"), assessment and diagnosis ("traumadiagnosis"), therapist self-care ("traumacare"), and therapy or other post-traumatic interventions ("traumatherapy" or "traumavention"). Each 
Section of the volume begins with an overview of goals to orient the reader to how subsequent materials will unfold, and ends with a self-assessment series of exercises, "Testing Your Expertise." In terms of traumadiversity and traumagenesis, the Sections of the volume deal with some of the most important problems facing clinicians in assessment, treatment, and research. The focus of Section I is on giving the reader a solid knowledge base in PTSD technology before issues of assessment and intervention are presented.

The Handbook/Manual thus begins with a comprehensive discussion of the nature and diversity of traumatic events, the epidemiological data on PTSD in various victim/survivor groups, and the multiplicity of psychobiological responses to traumatizing stimuli in victims. Also highlighted here are such issues as the impact of natural and technological disasters, long-range impact, and gender differences. There are illustrative examples on combat-related stress (Vietnam, Korean, Desert Storm, and Israeli war veterans, and children in war zones), on rape victims, victims of domestic abuse, victims of child sexual abuse, crime victims, a cautionary note on false memories, and caution on the use of stages/phases of emotional reactions over time. Four important Tables offer the reader a concise, easily accessible set of data on physiological alterations in PTSD, neurohumoral/neuroendocrinological abnormalities, DSM-IV Criteria for PTSD, and on Disorders of Extreme Stress (DES).

Other specific topics in this Section are: the relevance of identifying seven different classes of "clients," short-term vs. long-term traumatic events, vicarious exposure (and "compassion fatigue"), distinction between Type I and Type II traumas; post-traumatic stress response categories (i.e., emotional, cognitive, biological, behavioral and characterological); symptomatic responses (e.g., dissociation), the cardinal symptoms of PTSD, and a very illuminating and clinically enriching statement on phenomenological and narrative responses.

Acute Stress Disorder (ASD), a new diagnostic entity appearing in the DSM-IV, partial PTSD, Complex PTSD, and comorbid post-traumatic configurations (i.e., PTSD, and anxiety, panic attacks, depression, somatization disorder, alcohol abuse, compulsive gambling, borderline personality disorder, obsessive-compulsive symptoms, phobias, bulimia, anorexia, and compulsive overeating), are detailed for the reader. After each major subject in the many Sections of the volume, is a list of items titled, "What shall you take from the data on," for example, crime victims, child sexual abuse, etc.? This feature offers the reader a concise summary of PTSD information, and may be referred to as needed over time as a quick reference. The Section ends with "Testing Your Expertise"-a series of 20 clinical sce- 
narios to see how well the materials on the epidemiology/diagnosis of PTSD are understood, and the degree of achieved expertise.

Section II advances the author's views on conceptualizing PTSD. He chooses a "constructive narrative perspective" (CNP) that focuses on personal "accounts" or "stories." CNP has a very long history, with roots in the philosophical writings of Immanuel Kant, Ernst Cassirer, and Jean-Paul Sartre, and the psychological works of Wilhelm Wundt, Alfred Adler, George Kelly, Jean Piaget, Viktor Frankl, and Jerome Frank. A more common name for CNP is the constructivist perspective, where the human personality is said to be based on the personal meanings people create. Constructing meaning goes beyond merely reacting to what is seen, heard, or felt. It involves the individual's own interpretations of events. Dr. Meichenbaum captures the essence of CNP through such quotations as "We are the stories we tell" (McCabe and Peterson). From this perspective therapy is viewed as a means of assisting the victim-client to construct a new, post-traumatic narrative and world. Table 1 presents examples of the cognitive reframing of post-traumatic cognitions which the author integrates from the works of many well-known giants in the field of clinical psychotraumatology.

Useful lists of client-generated metaphors reflecting both their traumatic affective state, and their quest for psychological healing and social integration are also included here. For this Section nine items are used to test the reader's expertise on alternative conceptualizations of PTSD. This Section focuses on a multiplicity of traumaconcepts and traumadynamics.

Assessment strategies in Section III have two components and relates to traumadiagnosis: open-ended questions to help clients tell his or her story and then to rescript the narrative to create new behaviors, and the use of standardized measures of PTSD. In concert with this reviewer's experience and preference, the author espouses multiple approaches in the assessment processes with adults, children, adolescents, and families who suffer the aftereffects of a specific trauma incident. This calls for addressing both the client's symptoms and phenomenological experience, as well as the positive benefits that many survivors have acquired as a result of the traumatic experience. A detailed discussion of various factors that shape the nature of the assessment process, and a useful sequential format (suggested guide) consisting of Format I for comprehensive assessments and Format II for briefer ones. Both Formats are detailed with specific guiding statements.

The challenges presented by differential diagnosis and the determination of the role of premorbid factors are detailed here, and the question, "Who is at risk?" is answered completely for the reader. Comorbidity measures in terms of general psychopathology, depression, suicidal indicators, substance abuse are discussed in detail as well. 
Another clinically valuable feature found throughout the Handbook/Manual are places where the reader is given specific details of what the clinician may say to a client in improving the quality of the interaction, and to elicit vital data in a variety of areas. For example, the author has included a subsection titled, "What Questions Can the Clinician Use with PTSD Clients?" Here the author begins with the initial interview, then outlines eight different categories of interview questions to elicit specific kinds of clinical data. He mentions the BATHE technique recommended by Stuart and Lieberman (in their Fifteen Minute Hour) that saves clinical time and yet increases efficacy in conducting a complete and meaningful interview. Because most questions clinicians need to ask are chiefly of the What and How varieties, Dr. Meichenbaum includes a significant list of "What Questions" and "How Questions." These are important features which clinicians may use to maximize the positive effects of their clinical efforts in the shortest amount of time. He gives reasons clinicians should avoid "Why" questions as much as possible.

In this context the author discusses styles of questioning, suggests questions to help clients tell their story more fully, more successfully, while increasing the sense of personal control. By asking key questions and engaging the client in a Socratic encounter the client takes responsibility for the journey to recovery. This same format of questioning can be used to attain a rape history, to establish treatment goals, to gain important data in childhood sexual abuse cases, to reframe traumatic events, and to increase the client's coping.

Moreover, the author shows how to help clients take a different perspective, and become less reluctant or resistive to disclosing painful victimization data. To assist clients move toward a problem-solving mode, the author demonstrates how to help them define the problem, its severity and its generality, how to enable him or her to pin down the specific conditions that intensify and alleviate symptoms, as well as to identify the origins and antecedents of traumatic stress responses in their daily lives.

The author also crafts useful statements designed to produce desired outcomes regarding clients' perception about how the therapist sees things, how clients view the therapist, and items that increase collaboration and design a mutually workable agenda. Transaction statements are geared to nurture the therapeutic alliance, and to enhance the value of the therapeutic communication generated by transference and countertransference feelings by "going public with the data."

The role of standardized instrumentation in PTSD assessment is discussed as well as a number of specialized instruments and the specific symptoms these instruments purport to measure. For example, structured clinical interviews, clinical rating scales, combat-related self-report scales, and sexual 
and physical measures, as well as measures of domestic violence, child abuse, crime-related PTSD, schema-related measures (how trauma affects beliefs systems), social support, and measures of psychophysiological, psychoendocrine, and sleep disturbances, and of community factors, marital distress, coping, general adjustment, goal attainment, ethnic identification, and the challenge of differential diagnoses and comorbidity. A clinically sophisticated assessment of suicidal intention, behavior, and management is a very valuable contribution appearing in this Section. Specific details on how to assess PTSD in children and adolescents, cynical hostility, eating disorder, and substance abuse will prove useful to clinicians. The section ends with a 16-item "Testing Your Expertise" on assessment strategies and procedures.

In terms of traumacare, Section IV begins with an examination of the nature of "traumatic memories" and the importance of recognizing therapists' needs as helpers. Heatedly debated issues around "repressed memories" or "false memories," and caution about the "dangers that reside in the potential iatrogenic (therapist-initiated) influences on the assessment and therapeutic processes" are briefly discussed. Additionally, ways to manage vicarious traumatization and secondary victimization, depression, despair, cynicism, alienation, psychological and physical symptoms, and sense of vulnerability are critical considerations to contemporary trauma clinicians. Trauma therapists who need assistance for their occupation-related stress may seek interventions through personal, professional (to include peer support and supervision), and organizational resources.

Section V, "Treatment Alternatives" reviews and synthesizes the scientific and clinical works on clinical techniques, and on process and outcome variables. This Section, additionally, is a virtual gold mine containing stateof-the-art information on traumatherapy for the clinician and clinically-focused research scientist who need vital data reliably integrated on post-traumatic stress behavior theory, and therapy. In great detail the author analyses diverse techniques being employed in the contemporary treatment of PTSD. The specific treatment alternatives discussed are: pharmacological, crisis intervention, individual time-limited dynamic, time-limited trauma, individual behavior therapies to include systematic desensitization. Clinician and researchers who are interested in EMG biofeedback, EMDR, guided imagery-based interventions, cognitive-behavioral individual and group therapies, stress inoculation training, dialectical behavior therapy, and marathon group therapy will find this Section of particularly valuable to their theoretical acumen and therapeutic effectiveness. Among the other therapies presented in the Handbook/Manual are: cognitive processing therapy, sanctuary milieu treatment program, multifaceted outpatient intervention (Koach program), readjustment counseling intervention, 
group programs for women, Gestalt techniques, marital and family therapy, multifamily therapy, hypnosis, general skills multimodal training; community-wide and school-based interventions, sexual dysfunction therapy, crosscultural counseling, pastoral care, wilderness programs, and political action interventions are among the treatment techniques available to readers of this volume.

Some of the treatment techniques outlined are reviewed from a critical perspective, pointing out strengths and weaknesses and the need for future research. For example, exposure-based therapies, EMDR, and CISD are presented along with general evaluative comments. A significant number of specific treatment issues most clinician grapple with in their work are highlighted to enhance therapists' expertise. These include: How Long Should Therapy Take with PTSD Clients?, How to Create Concrete Representations of the Abuse?, reenactments of the abuse experience, affect tolerance, memory recall, stigmatization, powerlessness, sense of betrayal, tramagenic sexualization, and procedures for ending the cycle of repeated victimization.

In a subsection titled, "General Treatment Guidelines: What Can Be Done to Help Clients With PTSD and DES," the procedures begin with Phase I (Introduction) and progress to Phase V (Termination). This is a step-by-step model clinicians may use with confidence. Being consistent with his basic therapeutic premises, the author encourages therapists to be concerned not only with clients' restorative needs (to handle deficits) but with their constructive need for positive growth as well. After "Testing Your Expertise" on treatment alternatives, the volume moves to "Specific Treatment Procedures for Adults with PTSD and Related Problems."

Section VI begins with a systemic plan to educate the client about the nature of PTSD and the recovery process. This is done by teaching the client about the need to normalize and legitimize his or her reactions to the trauma, how to understand trauma responses from a biopsychosocial perspective, how to reframe symptoms, how to bolster client's hope, and how to recognize and prevent lapses and relapses. When interacting with the client the therapist is correctly admonished, "Do not act like a surrogate frontal lobe for your clients!" and to give instructions to clients in a way that is "specific, clear, detailed, concrete, and simple."

When treating clients with problematic flashbacks the author offers helpful suggestions, and elaborates on how to develop coping skills, alter troublesome memories, and on how to facilitate the processing and integrating of flashback phenomenon by observing the "messages" found in the flashbacks. Offering the clinician a plethora of specific tools to be employed in traumatherapy, the author discuses imaginal and in vivo exposure procedures (such as imaginal exposure and live exposure), guided imagery 
(e.g., adult-nurturing child imagery), cognitive processing therapy (CPT), use of hypnosis, and a very helpful Table that serves as a systematic guide in the form of a check list to be used in direct therapeutic exposure (DTE) and guided imagery-based interventions are described in detail.

For easy presentation and clinician-accessibility, the author discusses the powerful principles and proven techniques of stress inoculation training (SIT) by using a flow chart format, and a description of the various phases of SIT-conceptualization, skills acquisition, rehearsal, application, relapse prevention and follow-through. He also discusses issues of prevention, and moves on to help therapists help clients handle anger, rage, and dysphoric affects in a more effective manner. This is one of the most clinically enlightening aspect of this volume. Also, at the practitioner's fingertips are the specifics of cognitive restructuring procedures (examines cognitive events, cognitive processes, and cognitive structures to help clients overcome their automatic thoughts), imagery reconstruction, advice-giving, videotape, modeling film, self-monitoring, techniques on how clients can conduct personal experiments, and the value of letter-writing, audiotaping, and videotaping as therapeutic tools. After a 20-item "Testing your Expertise" exercise, the volume moves into Section VII, "Post-Disaster Interventions."

The Handbook/Manual details post-traumatic stress disaster-related responses, beginning with a brief overview of the disaster literature, followed by a discussion of relevant issues on assessment and diversity of risk factors in the development of PTSD. This Section also offers guidelines to traumavention, specific approaches to assist disaster victims. Interventions strategies illuminated in this Section are: critical incidents stress debriefing (CISD-its goals, format, and a critical evaluation); peer support, outreach model, debriefing children and families, school-based interventions, and military applications.

Additionally, a series of disaster relief guidebooks are introduced to the reader, and the role of rituals and religion are viewed as important considerations in traumatherapy in the aftermath of disasters. The Handbook/Manual shows clinicians, moreover, how to conduct a debriefing session. Additionally, several alternative post-trauma disaster models of intervention, postvention procedures and school-based interventions are presented in detail.

An epilogue ("Looking Into the Future of Clinical Training") follows the "Testing Your Expertise" on post-disaster interventions, and Section VIII begins. This Section is a client/consumer-oriented one, consisting of lists of resources available to victims who desire to know more about spousal battery, incest, child abuse, and crime, and other specific issues in the trauma literature. 
The book ends with a comprehensive set of references on PTSD theory, clinical phenomenology, and scientific studies pertaining to adults, children, and adolescents, as well as a list of references on psychometric assessments for PTSD, iatrogenesis and memory, alcoholism and addictions, and cognitive, motivational and social correlates of alcohol abuse.

The relevance of the Handbook/Manual to traumatherapy and to traumascience is quite evident. It offers the reader an integration of the clinical and scientific aspects of PTSD technology, focusing on extant theoriesbehavioral, cognitive, psychodynamic, phenomenologic-existential, as well as spiritual dimensions of healing after trauma.

In a field with such a large diversity of ideas scattered all across the entire clinical-scientific landscape, this volume brings the pieces together using the best available information to date. As master clinician, Dr. Meichenbaum offers a wealth of ideas and practical "how-to" procedures that are easily understood and applied. The clarity and specificity with which the volume's materials are displayed may conceivably stimulate research into theories and techniques still in need of scientific validation.

This book appeals to a very broad professional audience-psychologists, psychiatrists, other physicians, nurses, rehabilitation specialists, program administrators and directors traversing the gamut from beginner to advanced to expert levels.

The impact of the volume on the field of psychotraumatology promises to be significant because it is the only essential reference work on the phenomenological, theoretical, diagnostic, therapeutic, preventive, practical, consultative, and scientific aspects of recovery after biopsychic trauma. The volume's approach shows a high degree of congruence with my own theoretical and technical choices with trauma victims/survivors over almost two decades. It thus presents traumatherapy as a multitheoretical (conceptual), multiphasic (temporal), multidimensional (complexity), and multitechnical (strategy) enterprise (Parson, 1994). Hopefully, its contribution to the field will result in future efforts to employ multiple theories (from several schools of therapy), and to overcome a significant problem I see today in contemporary conceptualization of traumatherapy; namely, the exclusive overemphasis on single-theory approaches to treating victims with complex biopsychobehavioral impairments (Parson, 1994).

The readability of the volume may prove difficult to some were it to be read from cover to cover. This is because the Handbook/Manual is a 600-page volume, featuring over 500 headings and subheadings, eight Sections, a Prologue, Epilogue, and resources lists of almost 2,000 references! The author thus correctly suggests that readers use a procedure comparable to the "line-upon-line, word-upon-word" format for optimal value and utility. 
Dr. Meichenbaum includes "user-friendly" guides throughout the volume to assist the reader find all possible data pertaining to a particular topic. This may require searching within one Section or looking in multiple Sections. For example, if the reader desires information on rape victims, he or she will find the desired information by traversing across the pages and Sections of the volume. Large dividends are to be realized by readers who become familiar with the volume's layout and contents.

I therefore recommend the Handbook/Manual to any clinician, administrator, or scientist who has always wanted to know almost everything about psychological trauma-stressor characteristics, post-traumatic responses, and PTSD, and the assessment process and intervention strategies that work. This book should be required reading in any program that trains professionals in the complexities of biopsychobehavior stress responses and the intervention strategies to help victims/survivors of overwhelming life events.

\section{REFERENCE}

Parson, E. R. (1994). Post-traumatic stress disorder: Its biopsychobehavioral aspects and management. In B. Wolman and G. Stricker (Eds.), Anxiety and related disorders: $A$ handbook (pp. 226-285). New York: Wiley.

Erwin Randolph Parson, Ph.D.

Narcissistic Giving: A Study of People Who Cheat in Relationships. Gerald Alper. New York: International Scholars Publications, 1995.

Gerald Alper never writes dull books. A novelist and short story writer turned psychotherapist, he has one of the more creative analytic minds of his generation. A bit quirky, slightly askew, he puts a unique spin on the psychoanalytic understanding of human behavior. In his latest work, Narcissistic Giving: A Study of People Who Cheat in Relationships, Alper comes up with a host of new and sometimes jaundiced perspectives on relationships and what drives them. Alper is particularly fascinated by asymmetrical relationships in which inequalities of power are simultaneously denied and manipulated in such a way that the more powerful of the pair is able to apparently give something valuable to the weaker of the two while actually giving nothing. Alper's "narcissistic giver", a category and phrase which may be destined to enter the psyche and vocabulary of the psychologically literate, differs from the "Santa Claus narcissist", who actually gives much 
in service of aggrandizing his or here own ego, in giving nothing-it's all a con. Alper spins out the dialectical interplay between narcissistic giver and victim with virtuosic skill, drawing the reader into the maze of deception and self-deception, bafflement and rage, confusion and depression that the recipient of acts of narcissistic giving suffers. When I started the hook I wondered if Narcissistic Giving might not itself be an act of narcissistic giving-the idea seemed too slight and once brought to one's attention not sufficiently remarkable to carry a book length essay-but by the time Alper was finished his many layered I phenomenological, dynamic, sociological, political, and aesthetic-permutations on his basic theme, I felt that I had indeed received a valuable gift-insight into the pervasiveness of a pernicious mode of relating at every level from the interpersonal to the societal and a deepened awareness of a form of emotional devastation that is often so smooth that the sufferer has no idea of what happened to him or her.

All of Alper's ongoing concerns are here: the relationship of artist and audience, the enemies of paranoids, the struggles of the young for recognition, the subtleties of victimization, the abuse of power by institutions and individuals in the mental health field, and the interplay of the psychological and the sociological. Filled with memorable phrases such as "tangential intimacy", "fail-safe relating", "objective mania", and "action relationships" Narcissistic Giving moves from the Hegelian to the Laingian without pretension. Although I sometimes wished for more narrative and less discussion, this is an enjoyable, insightful, fluent, and original work which speaks to both the professional therapist and the general reader.

Jerome David Levin, Ph.D.

Right Side Up! Reflections for Those Living with Serious Illness. Marlene Halpin, Ph.D. Dubuque, Iowa: Islewest Publishing, 1995.

In 1984, at a retreat at Holiday Hills in New York I had the rewarding experience of attending a four day seminar with Marlene Halpin as the leader. The title was "Imagination: Its Place and Power in Adult Ministries." I was enthralled by the use of guided imagery in self exploration and self understanding. This began a journey I am still on. I immediately purchased her book "Imagine That: Using Phantasy in Spiritual Direction." I certainly would recommend this to anyone wanting to know more about the effective use of guided imagery techniques.

When I recently heard that she had written another book I couldn't wait for the opportunity to read it. This book is the one being reviewed 
at this time-Right Side Up! Reflections for Those Living with Serious IIlness. It is, to my surprise, quite a different book from the first, but yet it is a continuation of it in some respects. It is a book of listening, one that documents, in the form of poetry, the experiences of many people who suffer from a variety of illnesses, and of those who care for them. In studies of death and dying, loss and grief, many are the textbooks, but few reverberate with the suffering, touching on all the feelings. All who work with human suffering should read this book. "Very ill people think and feel a great deal," a hospice nurse observes, "but much of it does not get communicated at all because....." (p. v). The reasons are many, but mainly because others cannot hear. The author of Right Side Up! demonstrates that she can hear. And, upon hearing, she can reflect. And, having reflected, she puts the feelings into poetry and art.

If one already has a grasp of the stages of dying, yet has trouble grasping the serenity of acceptance that is the goal of the process, then this book should help. This will move the concepts from left to right brain, from the irrational to the emotive. If one cannot operate on the intuitive level, then work among people with serious illnesses will be difficult indeed. The book instructs in a non-teaching way. If one can read this book slowly and quietly, and not be touched and benefit from it, then I would suggest a reevaluation of one's professional role with people who suffer conditions that generate persistent pain and anguish. Perhaps in today's hectic life, a book that calls for careful, thoughtful, and repeated readings may seem impractical to many, but I see no alternative. This book touches every emotion one feels when working with loss and grief.

In addition to the volumes instructors usually recommend to their students, I would hope each classroom instructor, and each loss and grief workshop leader require each student or participant to have this book as required reading. In the book the author invites individuals with serious illnesses, and those who care for them to send their poems, prayers, or reflections about what it's like to have the experiences they endure. This would be an excellent learning objective, for if we are unable to know the suffering intuitively, how shall we help effectively?

David W. Pierce, M. Div. 